\title{
Wearable exoskeleton control modes selected during overground walking affect muscle synergies in adults with a chronic incomplete spinal cord injury
}

\author{
Manuel J. Escalona $\mathbb{1}^{1,2} \cdot$ Daniel Bourbonnais ${ }^{1,2} \cdot$ Michel Goyette $\mathbb{I}^{2} \cdot$ Cyril Duclos $\mathbb{D}^{1,2} \cdot$ Dany H. Gagnon ${ }^{1,2}$
}

Received: 28 January 2020 / Revised: 9 March 2020 / Accepted: 10 March 2020

(c) The Author(s), under exclusive licence to International Spinal Cord Society 2020

\begin{abstract}
Study design Case series.

Background Changes in the number of muscle synergies (MSs) and in the weighting of muscles composing each MS are typically altered following an incomplete spinal cord injury (iSCI). Wearable robotic exoskeletons (WRE) represent a promising rehabilitation option, though the effects of various WRE control modes on MSs still remain unknown.

Objective This case series characterizes how WRE control modes affect the number of MSs and the weighting of muscles composing each MS in individuals with iSCI.

Setting Pathokinesioly laboratory of a rehabilitation research center.

Methods Three participants with a chronic iSCI walked at a self-selected comfortable speed without and with a WRE set in two trajectory-controlled (Total Assistance, TOT; Assistance-as-Needed, ADAPT) and three non-trajectory controlled modes (High Assistance, HASSIST; High Resistance, HRESIST; NEUTRAL). Surface EMG of eight lower extremity (L/E) muscles was recorded and used to extract MSs using a nonnegative matrix factorization algorithm. Cosine similarity and weighting relative differences characterized similarities in MSs between individuals with iSCI and able-bodied controls. Results The mode providing movement assistance within a self-selected L/E trajectory (HASSIST) best replicated MSs in able-bodied controls during overground walking. MSs extracted with the trajectory-controlled modes differed to the greatest extent from able-bodied group MSs.

Conclusions Most WRE control modes did not replicate the motor control required for typical L/E muscle coordination during stereotypical overground walking. These results highlight the need to gain a better understanding of the effects of various control modes on $\mathrm{L} / \mathrm{E}$ motor control for rehabilitation professionals to incorporate research evidence when selecting WRE control mode(s) during WRE locomotor interventions.
\end{abstract}

\footnotetext{
* Part of this work was presented during the 26th annual conference of the Société Francophone Posture Équilibre et Locomotion (December 4th and 5th, 2019, Montreal, Canada) and published in the form of an abstract.
}

\section{Dany H. Gagnon \\ dany.gagnon.2@umontreal.ca}

1 School of Rehabilitation, Faculty of Medicine, Université de Montréal, Montreal, QC, Canada

2 Pathokinesiology Laboratory, Center for Interdisciplinary Research in Rehabilitation of Greater Montreal (CRIR), Institut universitaire sur la readaptation en deficience physique de Montreal, CIUSSS Centre-Sud-de-l'Ile-de-Montréal, Montreal, QC, Canada

\section{Introduction}

Overground locomotor training with Wearable robotic exoskeletons (WRE) represents an emerging and promising neurorehabilitation intervention that aligns with the basic principles of motor learning (e.g., specificity, repetition, and intensity) promoted after a neurological lesion [1]. However, it still remains difficult to pinpoint how this intervention compares with conventional locomotor training interventions in adults with an incomplete spinal cord injury (iSCI) [2-4]. Part of this difficulty relates to the fact that almost all evidence have been gathered using WRE with total lower extremity (L/E) motorized assistance and fixed trajectory guidance during treadmill walking. As a result, after having gained sufficient experience with the WRE, active voluntary participation and stride-to-stride variability, 
which are essential components in motor learning, becomes regulated and may negatively affect walking recovery [2]. In fact, such an approach may induce a habituation and sensitization phenomenon in which the spinal cord circuits adjust rapidly to repetitive activations of the same sensory pathways [5].

To overcome these obstacles, while also increasing perceived utility and acceptability among rehabilitation professionals, some WRE manufacturers offer L/E control modes providing various levels of assistance or resistance, as well as non-imposed (i.e., non-controlled) trajectory guidance. In the neurorehabilitation context, these $\mathrm{L} / \mathrm{E}$ control modes allow rehabilitation professionals to personalize WRE-based rehabilitation interventions to maximize locomotion and locomotion-related abilities. However, the effects of the various control modes on L/E muscle coordination underlying locomotion remain unknown, and clinical practice remains predominantly informed by clinical reasoning and accumulated experience.

The L/E muscle coordination can be revealed by characterizing muscle synergies (MSs) using nonnegative matrix factorization (NNMF) algorithms. This analysis usually reveals a specific number of MSs (i.e., motor modules) with muscle weightings associated with gait subcycles. In adults with iSCI, spinal locomotor control is compromised to various extents and consequently alters how the central nervous system (CNS) coordinates the muscles involved during locomotion. This generally translates into fewer L/E MSs, or an altered weighting of the different muscles involved in a given MS leading to motor impairments during gait [6]. Thus, increasing the number of MSs, or replicating the weighting of muscles similar to those synergies found in able-bodied individuals during overground locomotor training with a WRE, could theoretically translate into improved walking abilities in individuals with iSCI. However, to our knowledge, no study to date has investigated to what extent various WRE control modes may modify the number of MSs and the weighting of muscles composing each MS during overground walking with a WRE in individuals with iSCI who have recovered to various extents their ability to walk.

The aim of this case series is to examine how various WRE trajectory and non-trajectory control modes affect L/E MSs (e.g., number of MSs, weightings of muscle within a given synergy) in individuals with iSCI during overground walking with a WRE. It is hypothesized that the number of MSs and weighting of muscles composing each MS during overground walking without WRE will differ when compared with able-bodied MSs (H1). Moreover, walking with a WRE set in a non-controlled trajectory mode will increase the number of MSs and modify weightings of muscles composing each MS to levels comparable to those extracted in able-bodied individuals (H2). This research represents an initial step to strengthen evidence regarding $\mathrm{L} / \mathrm{E}$ muscular coordination that will inform clinical practice on the effects of different control modes when planning personalized WRE locomotor interventions.

\section{Methods}

\section{Participants}

Three participants with traumatic chronic iSCI (ASIA Impairment Scale, AIS $=\mathrm{C}$ or D) below the fifth cervical neurological level were recruited for this study. Participants were included if they were able to walk overground for at least $10 \mathrm{~m}$ without or with a walking aid (e.g., forearm crutches); were able to follow verbal, visual, and auditory commands; and met all WRE manufacturer requirements (e.g., L/E passive range of motion limitations, moderate-to severe L/E spasticity) as verified by a comprehensive physical therapy assessment. Participants were excluded if they presented history of other neurological disorders, including nontraumatic SCI or cognitive impairments. The study was conducted at the Pathokinesiology Laboratory located at the Institut universitaire sur la réadaptation en déficience physique de Montréal. All participants provided written consent to participate. The Research Ethics Committee of the Center for Interdisciplinary Research in Rehabilitation of Greater Montreal (CRIR) approved the study (CRIR-1083-0515). All applicable institutional and governmental regulations concerning the ethical use of human volunteers were followed during the course of this research.

\section{Clinical evaluations}

Injury severity was evaluated by a certified physiotherapist using the American Spinal Injury Association Impairment Scale (AIS) to categorized participant's neurological injury level and completeness. The L/E muscle strength was assessed and graded according to the Lower Extremity Motor Score (LEMS) of the International Standards for Neurological Classification of SCI. The $10 \mathrm{~m}$ walking test was completed at self-selected natural velocity to evaluate walking speed and confirm the participant's ability to walk the test distance.

\section{Robotic exoskeleton}

The Ekso GT ${ }^{\mathrm{TM}}$ WRE (EKSO Bionics, CA, USA) provides robotic control during overground walking. Specifically during the swing phase, the control modes offered by the Ekso $\mathrm{GT}^{\mathrm{TM}}$ can be grouped into trajectory control, including total assistance (TOT) and assistance-as-needed 
(ADAPT) modes, as well as non-trajectory control, including high assistance (HASSIST), high resistance (HRESIST), and NEUTRAL modes (Fig. 1). During stance, knee flexion beyond 45 degrees was blocked by the WRE to prevent full knee collapse and falling.

\section{Intervention}

Participants completed four 45-60 min training sessions over a 2-week period. During these sessions, under direct supervision of a certified physiotherapist, participants learned to safely walk with the WRE at a self-selected comfortable speed using forearm crutches and with the WRE set in the five WRE control modes along a $50 \mathrm{~m}$ level tiled corridor.

\section{Laboratory assessment}

\section{Walking conditions}

Participants walked without the WRE at a self-selected natural speed (NAT) on a leveled tiled corridor over a $10 \mathrm{~m}$ distance. Thereafter, participants walked with the WRE at a self-selected comfortable speed with all WRE control modes tested in a random order (i.e., participant 1: HASSIST-ADAPT-NEUTRAL-HRESIST-TOT; participant 2: NEUTRAL-ADAPT-HRESIST-TOT-HASSIST; participant 3: HRESIST-HASSIST-ADAPT-NEUTRAL-TOT). Immediately after testing each control mode, the participant's rate of perceived exertion (RPE) was collected using a modified 0-10 Borg Scale. Between modes, participants performed lateral weight shift transfers while standing for $1 \mathrm{~min}$ to minimize any potential carryover effects of the previously tested WRE mode (i.e., wash out).

\section{Surface electromyography}

Using a Delsys Trigno wireless EMG system (Delsys Inc., Boston, MA, USA), the EMG activity was recorded from eight L/E muscles bilaterally: gluteus medius (GM), rectus femoris (RF), vastus medialis (VM), semitendinosus (ST), biceps femoris (BF), tibialis anterior (TA), medial gastrocnemius (MG), and soleus (SO). After proper skin preparation, all wireless hybrid sensors were positioned in accordance with recommendations of the Surface ElectroMyoGraphy for the Non-Invasive Assessment of Muscles (SENIAM) (www.seniam.org) to enable surface EMG $(1926 \mathrm{~Hz})$ and 3D acceleration data $(148 \mathrm{~Hz})$ recording.

Raw EMG data were filtered (Butterworth bandpass $20-400 \mathrm{~Hz}, 4$ th order no lag) and processed with a continuous Root Mean Square (RMS) using a centered $250 \mathrm{msec}$ moving window. Each gait cycle was delimited between consecutive foot contacts, which were determined from integrated acceleration peaks from the SO sensors using a Teager-Kaiser Energy Operator (TKEO), and then visually inspected and manually adjusted if needed. All gait cycles were time normalized to $100 \%$ with $1 \%$ increments from which the stance (0-59\%) and swing (60-100\%) phases were depicted. For each walking condition, the best three consecutive cycles,

1. Trajectory controlled: The wearable exoskeleton (WRE) automatically initiates steps when the participant reaches both predetermined lateral and forward body shift thresholds. Once the step is initiated, the exoskeleton swings and controls the hip and knee kinematics for the foot to follow a specific pathway.

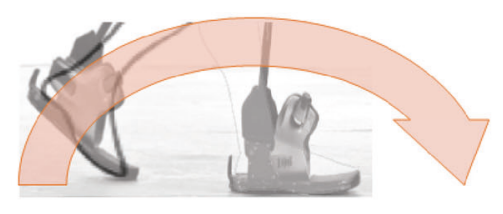

\begin{tabular}{cl} 
1.a. TOT & $\begin{array}{l}\text { Provides total motorized assistance continuously to move the hip } \\
\text { and knee joints according to a predefined planned hip and knee } \\
\text { kinematics configured to drive foot position during the swing } \\
\text { phase. }\end{array}$ \\
\hline 1.b. ADAPT & $\begin{array}{l}\text { Provides adaptable motorized assistance to continuously adjust hip } \\
\text { and knee joint movements to comply with a predefined planned } \\
\text { hip and knee kinematics configured to drive foot position during } \\
\text { swing phase. }\end{array}$
\end{tabular}

2. Non-trajectory controlled (i.e., 'free legs'): The participant initiates swing and control freely his L/E kinematics (amplitude, velocity, and acceleration) within the sagittal plane (i.e., no predefined trajectory) during the swing phase of each step.

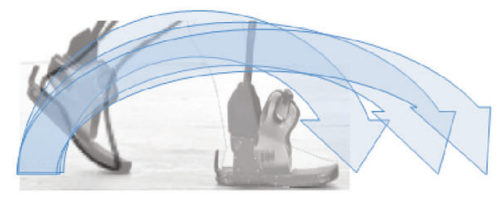

\begin{tabular}{cl} 
2.a. NEUTRAL & $\begin{array}{l}\text { Provides no assistance and no resistance at the hip and knee joints } \\
\text { during swing phase. Only gravity compensation assistance is } \\
\text { provided. }\end{array}$ \\
\hline 2.b. HASSIST & $\begin{array}{l}\text { Provides high assistance to facilitate hip flexion and knee } \\
\text { extension during swing phase. }\end{array}$ \\
\hline 2.c. HRESIST & $\begin{array}{l}\text { Provides high resistance to augment hip flexor and knee extensor } \\
\text { muscular efforts during swing phase. }\end{array}$
\end{tabular}

Fig. 1 Description of the different exoskeleton control modes investigated during the swing phase when walking with the wearable robotic exoskeleton. 
based on the lowest mean coefficient of variation computed for all EMG envelopes over each temporal data point embedded within each time normalized cycles, were automatically selected using a custom-made Labview software before being averaged and amplitude-normalized (i.e., the RMS from each muscle was divided by its own maximum peak value prior to initiating the MSs analysis).

\section{Muscle synergies}

An experimental EMG data matrix was calculated for each participant, consisting of the mean of three consecutive gait cycles of each recorded muscle, prior to being submitted to a NNMF algorithm. The number of MSs was determined by the least number of synergies that could explain the variance accounted for (VAF) in each muscle $\left(V A F_{m}\right)$, with $V A F_{m}>0.9(90 \%)$ and the product of all $V A F_{m}$ (global VAF, $\left.V A F_{g}\right)>0.8(80 \%)$. MSs were grouped based on the Cosine Similarity (CS) of the weight matrices (W) $[7,8]$. To analyze the resemblance between the obtained MSs of each walking condition against reference MSs computed among an able-bodied control, CS was calculated between each participant MSs (Wr) against those obtained from a reference participant [9]. The reference MSs were extracted from an able-bodied participant (i.e., control) who was assessed during overground walking without the exoskeleton using the same experimental protocol (i.e., equipment, recorded muscles, and experimental conditions) [10]. For this analysis, the inner product of the obtained MSs on each walking trial was calculated and the cosine angle between those synergies and the reference MSs was measured.

According to the reference able-bodied control, the muscles composing each MS were established as: Synergy \#1, GM, VM and to a lesser extent, RF; Synergy \#2, SO and MG; Synergy \#3, TA and RF; Synergy \#4, ST and $\mathrm{BF}$. The CS values closer to one indicated greater similarities in the directions of the two compared vectors. When the CS between Wr and Wt was $>0.868$ and statistically significant $(p<0.05)$ [11], MSs were considered similar. Whenever two distinct MSs in the same walking trial were classified into the same group, these two synergies were considered to have merged together. The synergy with the lowest correlation of the two was deemed to be merged to the synergy presenting the highest correlation value. Synergies not corresponding to any of the reference MSs extracted in able-bodied control were defined as "undefined".

To further visualize how each recorded muscle weighting contributing to a specific synergy was similar to those found in able-bodied reference, the weighting differences $(\mathrm{Wd}=\mathrm{Wt}-\mathrm{Wr})$ for each muscle and walking trial, were calculated for each participant (Fig. 2). In order to calculate $\mathrm{Wd}$, muscles belonging to a specific synergy were weighted by multiplying each muscle in the weight matrix by its maximum peak value found to obtained normalized values to one within each synergy and allow weighting matrices subtractions.

\section{Statistical analyses and interpretation}

Differences in MS weightings (Wd) equal to 0 represented perfect matches while values closer to 1 indicated larger divergences in MS weighting, with values ranging between 0 and 0.3 [12] considered to closely reproduce MSs weightings from the able-bodied control. The RPE values were calculated and interpreted according to the American College of Sports Medicine (ACSM) guidelines for exercise testing and prescription to determine the exercise intensity achieved while walking with the WRE [13, 14]. According to these guidelines, an RPE of 1-2 corresponds to very light to light intensity, 3-4 corresponds to a moderate intensity, 5-6 corresponds to a high intensity, and 7-10 corresponds to a very high intensity.
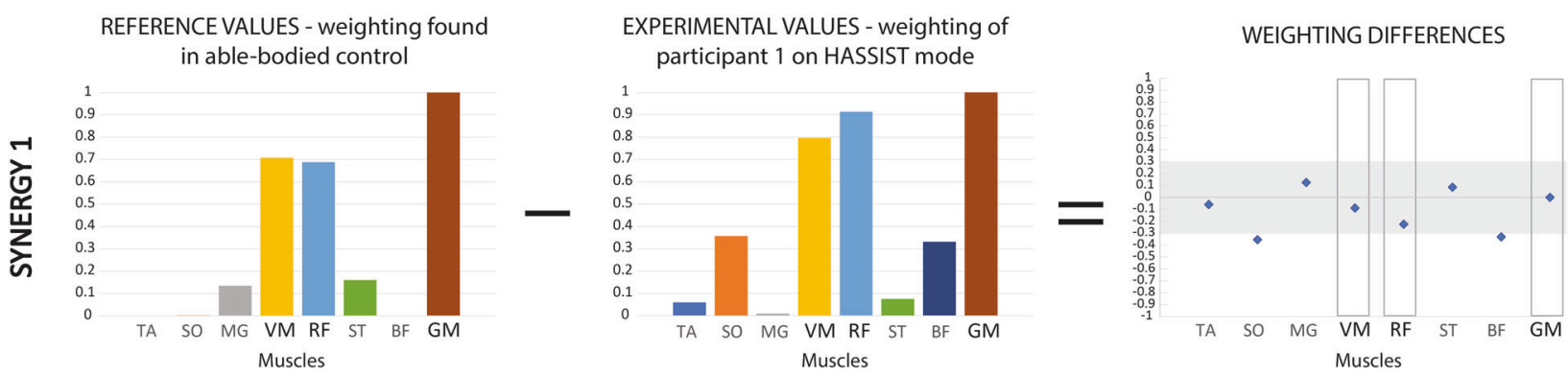

Fig. 2 Example of the procedure to calculate muscle weighting differences. Normal weighting matrix obtained on healthy individuals were subtracted to the obtained experimental tasks. The vertical gray boxes represent main muscles composing a specific muscle synergy while horizontal gray boxes represent the threshold range, i.e., $\leq 0.3$ for a value to be considered similar or close-to normal compared with a healthy reference. In this example, notice that synergy \#1, mainly composed by VM, RF, and GM muscles, presented almost perfect similarities with the HASSIST mode for participant 1 (color figure online). 


\section{Results}

\section{Participants and walking speed}

All demographic, clinical characteristics, and walking speed during each experimental trial are summarized in Table 1. Overall, compared with overground walking without WRE (i.e., NAT), walking with the WRE reduced speed between -63.2 and $-78.2 \%$ for participant 2 and between -55.6 and $-66.7 \%$ for participant 3, across all WRE control modes. Participant 1 predominantly walked faster than NAT by up to $42.1 \%$.

\section{Number of muscle synergies}

Three to four MSs were found across walking conditions (VAF $>0.8$ for all conditions). Synergy \#4 was absent in all participants with iSCI during NAT condition but present during all WRE control modes, except for participant 1 who had three synergies in TOT mode. Merging of MSs were observed and mostly found between synergies \#1 and \#4. Interestingly, only HASSIST mode consistently had all four

Table 1 Demographic and clinical information of participants.

\begin{tabular}{|c|c|c|c|c|c|c|}
\hline Participants & \multicolumn{2}{|l|}{1} & \multicolumn{2}{|l|}{2} & \multicolumn{2}{|l|}{3} \\
\hline \multicolumn{7}{|l|}{ Demographic characteristics } \\
\hline Gender & \multicolumn{2}{|l|}{ M } & \multicolumn{2}{|l|}{$\mathrm{F}$} & \multicolumn{2}{|l|}{ M } \\
\hline Age (years) & \multicolumn{2}{|l|}{42} & \multicolumn{2}{|c|}{51} & \multicolumn{2}{|c|}{60} \\
\hline Height (m) & \multicolumn{2}{|l|}{1.80} & \multicolumn{2}{|c|}{1.62} & \multicolumn{2}{|c|}{1.60} \\
\hline Weight (Kg) & \multicolumn{2}{|l|}{65.7} & \multicolumn{2}{|c|}{52.1} & \multicolumn{2}{|c|}{56.6} \\
\hline \multicolumn{7}{|l|}{ Clinical Information } \\
\hline Time since injury (years) & \multicolumn{2}{|l|}{18} & \multicolumn{2}{|c|}{1.1} & \multicolumn{2}{|c|}{40.7} \\
\hline Neurological level of injury & \multicolumn{2}{|l|}{ T6-T7 } & \multicolumn{2}{|c|}{$\mathrm{C} 5$} & \multicolumn{2}{|c|}{$\mathrm{T} 4$} \\
\hline $\begin{array}{l}\text { American Spinal Injury } \\
\text { Association Impairment } \\
\text { Scale (AIS) }\end{array}$ & \multicolumn{2}{|l|}{$\begin{array}{l}\text { D } \\
\text { (Trauma) }\end{array}$} & \multicolumn{2}{|c|}{$\begin{array}{l}\text { D } \\
\text { (Trauma) }\end{array}$} & \multicolumn{2}{|c|}{$\begin{array}{l}\text { D } \\
\text { (Trauma) }\end{array}$} \\
\hline Sensory level and score $/ 224$ & \multicolumn{2}{|l|}{ T7 197/224 } & \multicolumn{2}{|c|}{$\begin{array}{l}\text { C6 132/ } \\
224\end{array}$} & \multicolumn{2}{|c|}{$\begin{array}{l}\text { T4 156/ } \\
224\end{array}$} \\
\hline \multirow{2}{*}{$\begin{array}{l}\text { Total Lower Extremity Motor } \\
\text { Score (LEMS) }\end{array}$} & \multicolumn{2}{|l|}{$36 / 50$} & \multicolumn{2}{|c|}{$41 / 50$} & 46 & 150 \\
\hline & $R$ & $L$ & $R$ & $L$ & $R$ & $L$ \\
\hline Hip flexors & 4 & 3 & 5 & 5 & 3 & 5 \\
\hline Knee extensors & 2 & 3 & 5 & 5 & 5 & 5 \\
\hline Ankle dorsiflexiors & 4 & 4 & 4 & 4 & 5 & 5 \\
\hline Long toe extensors & 4 & 4 & 4 & 4 & 3 & 5 \\
\hline Ankle plantar flexors & 4 & 4 & 2 & 3 & 5 & 5 \\
\hline Walking speeds $(\mathrm{m} / \mathrm{s})$ & & & & & & \\
\hline Walking conditions & & & & & & \\
\hline Without Exoskeleton & NAT & 0.19 & & 0.87 & & 0.54 \\
\hline & Control modes & & & & & \\
\hline & TOT & 0.18 & & 0.19 & & 0.21 \\
\hline & ADAPT & 0.20 & & 0.25 & & 0.24 \\
\hline With Exoskeleton & NEUTRAL & 0.27 & & 0.28 & & 0.23 \\
\hline & HASSIST & 0.25 & & 0.26 & & 0.23 \\
\hline & HRESIST & 0.23 & & 0.32 & & 0.18 \\
\hline
\end{tabular}

$R$ right, $L$ left. synergies in able-bodied reference with relatively high CS values and no merged synergies. Undefined MSs were also found in most WRE control modes, except for HASSIST, which presented only one undefined synergy across all participants (Table 2).

\section{Muscles synergy weightings}

Table 2 illustrates similarities between weight matrices for each synergy and for each participant compared with an ablebodied reference using CS values. MS weightings during NAT varied widely across participants, particularly between the left and right L/E. The Wd analysis showed that TOT and ADAPT modes had very different patterns from the reference muscle weightings, illustrated by the scattered data point patterns of muscle weighting relative differences across participants and across MSs presented in Fig. 3.

Although some MSs weighting relative differences approached the 0.3 value threshold across the WRE control modes, only HASSIST consistently presented a clustering of point values around the $30 \%$ threshold on all participants and on both L/E. This confirms strong similarities between the HASSIST mode and the typical weight of muscles composing each MS found in the able-bodied reference.

\section{Rate perception of effort (RPE)}

The RPE across the different walking conditions are summarized in Fig. 4. Participants perceived effort levels ranging from light to moderate, with the greatest effort in HRESIST mode.

\section{Discussion}

The present study investigated the effects of different WRE control modes on MSs during walking in individuals with a chronic iSCI. To our knowledge, this is the first study investigating differences in MSs across a range of WRE modes. The three participants presented different degrees of sensorimotor impairment and functional disabilities resulting from their iSCI. The high variability across MSs attributes found across participants highlight the heterogeneity of muscle coordination challenges in adults with iSCI. Reduction in the number of MSs has been associated with an increased muscle co-contraction, poor muscle strength, or restricted joint range of motion because the CNS cannot independently and efficiently access and activate MSs during walking [6, 15, 16]. Clinically, these MSs deficits typically translate into abnormal motor outputs, decreased walking speeds, and increased gait asymmetry [6]. Thus, the reductions in the 
Table 2 Cosine similarities for all participants and walking trials. Notice that HASSIST mode presented four synergies with relatively high cosine similarities values, no merged and only one undefined synergy (values in italic).

\begin{tabular}{|c|c|c|c|c|c|c|c|c|c|c|c|c|}
\hline \multirow{5}{*}{ Participants } & & & \multicolumn{10}{|c|}{ Walking conditions } \\
\hline & \multicolumn{2}{|c|}{$\begin{array}{l}\text { Without } \\
\text { exoskeleton }\end{array}$} & \multicolumn{10}{|c|}{ With exoskeleton } \\
\hline & & & \multicolumn{10}{|c|}{ Control modes } \\
\hline & \multicolumn{2}{|l|}{ NAT } & \multicolumn{2}{|l|}{ TOT } & \multicolumn{2}{|c|}{ ADAPT } & \multicolumn{2}{|c|}{ NEUTRAL } & \multicolumn{2}{|c|}{ HASSIST } & \multicolumn{2}{|c|}{ HRESIST } \\
\hline & $R$ & $L$ & $R$ & $L$ & $R$ & $L$ & $R$ & $L$ & $R$ & $L$ & $R$ & $L$ \\
\hline \multicolumn{13}{|l|}{ Synergy 1} \\
\hline $\mathrm{P} 1$ & 0.71 & 0.68 & 0.88 & 0.78 & 0.91 & $\sim \# 4$ & 0.78 & $\sim \# 4$ & 0.94 & 0.92 & 0.78 & $\sim \# 4$ \\
\hline $\mathrm{P} 2$ & 0.76 & 0.81 & 0.73 & $\sim \# 3$ & 0.63 & $\sim \# 3$ & 0.75 & $\sim \# 4$ & 0.85 & 0.81 & 0.8 & 0.69 \\
\hline $\mathrm{P} 3$ & 0.83 & 0.76 & $\sim \# 4$ & $\sim \# 4$ & $\sim \# 4$ & 0.56 & 0.63 & 0.64 & 0.83 & 0.82 & 0.76 & 0.63 \\
\hline \multicolumn{13}{|l|}{ Synergy 2} \\
\hline $\mathrm{P} 1$ & 0.69 & 0.81 & 0.81 & & 0.79 & 0.8 & 0.74 & 0.83 & 0.75 & 0.83 & 0.74 & 0.92 \\
\hline $\mathrm{P} 2$ & 0.85 & 0.75 & 0.82 & 0.78 & 0.81 & $\sim \# 4$ & 0.8 & 0.92 & 0.9 & 0.91 & 0.94 & 0.85 \\
\hline $\mathrm{P} 3$ & 0.82 & 0.86 & 0.97 & 0.74 & 0.91 & 0.75 & 0.94 & $\sim \# 4$ & 0.84 & 0.81 & 0.92 & 0.83 \\
\hline \multicolumn{13}{|l|}{ Synergy 3} \\
\hline $\mathrm{P} 1$ & 0.87 & 0.67 & $\sim \# 2$ & 0.67 & $\sim \# 1$ & 0.82 & $\sim \# 4$ & 0.79 & 0.78 & 0.77 & $\sim \# 1$ & 0.8 \\
\hline $\mathrm{P} 2$ & 0.91 & 0.92 & 0.96 & 0.92 & 0.94 & 0.87 & 0.9 & 0.91 & 0.95 & 0.95 & 0.88 & 0.93 \\
\hline $\mathrm{P} 3$ & 0.84 & 0.86 & 0.85 & 0.92 & 0.94 & 0.87 & 0.84 & 0.91 & 0.89 & 0.88 & 0.83 & 0.87 \\
\hline \multicolumn{13}{|l|}{ Synergy 4} \\
\hline $\mathrm{P} 1$ & & & 0.94 & 0.82 & 0.91 & 0.8 & 0.81 & 0.8 & 0.9 & 0.87 & 0.8 & 0.68 \\
\hline $\mathrm{P} 2$ & & & 0.69 & 0.86 & 0.68 & 0.7 & 0.87 & 0.87 & 0.92 & 0.93 & 0.89 & 0.88 \\
\hline P3 & & & 0.86 & 0.84 & 0.88 & 0.85 & 0.78 & 0.71 & 0.87 & 0.78 & 0.77 & 0.84 \\
\hline \multicolumn{13}{|l|}{ UD Synergy } \\
\hline $\mathrm{P} 1$ & & & $X$ & & $X$ & $X$ & $X$ & $X$ & & & $X$ & $X$ \\
\hline $\mathrm{P} 2$ & & & & $X$ & & $\mathrm{XX}$ & & $X$ & & & & $X$ \\
\hline P3 & & & $X$ & $X$ & $X$ & $X$ & & $X$ & & $X$ & $\mathrm{X}$ & $X$ \\
\hline
\end{tabular}

$\sim \# \mathrm{X}=$ synergy merged with synergy number $\mathrm{X}$. number of MSs and altered muscle weighting within each MS were expected and fully support the first hypothesis (H1) that the number and weighting of muscles composing each MS during overground walking without WRE will be different than able-bodied MSs.

Synergy \#4 emerged while walking with the WRE in all control modes, with varying muscle weightings across participants and L/E sides. Although more MSs reflects improved motor function [17], the results prove otherwise since the more synergies during walking with the WRE did not necessarily match the typical weighting of muscles composing each MS. Indeed, most MSs weightings across control modes were different from those found in ablebodied controls during overground self-selected natural speeds. These findings indicate that, even during TOT and ADAPT control modes during which the L/E trajectory remains totally guided through typical, strict, and repetitive kinematics patterns [1], adaptations to these modes did not lead to a typical muscle activation pattern in adults with a chronic iSCI. This is of great relevance because TOT or ADAPT control modes, which are the most commonly used modes in the literature to explore superiority effects of robotic exoskeleton over other conventional locomotor training interventions in adults with iSCI [2], might not reinforce an adequate neural locomotor pattern for locomotion.

A key finding of this study, which partially supports the second hypothesis (H2), is that only HASSIST mode consistently replicated the number of MSs and weight of muscles composing each MS in the reference able-bodied individual. These findings were observed in both L/Es among all participants and in all main and secondary muscles composing each MS. This is demonstrated by the relative difference values below the $30 \%$ threshold set to establish similarities with able-bodied controls. Such effect of the HASSIST mode may result from an increased step variability triggered by the free/non-imposed L/E trajectory while allowing voluntary motor control, providing the assistance that may reduce the need of compensation or activation from other muscles that normally would not participate in a typical synergy. These elements might facilitate the recruitment of MSs similar to those found during overground walking in healthy individuals. Concerning NEUTRAL and HRESIST modes, this MSs 




weighting "normalization" was not achieved since the lack of assistance and increased limb motion resistance, alongside potential under-optimal compensations for the dynamics of the WRE, might have increased the probability of recruiting additional secondary/compensatory muscles to adapt to the new demands, which translated 
Fig. 3 Right and left muscle synergies weightings relative differences for all experimental trials and for each participant. The vertical gray boxes highlight the muscles defining a specific muscle synergy (i.e., the muscles that contribute the most on a synergy in heathy individuals). The light gray horizontal band represents the limits of $30 \%$ within which the synergies are found to be similar to those found in healthy individuals. Notice that for all synergies, the HASSIST mode consistently tended to bring the muscle weightings closer to $30 \%$ in all participants. TA tibialis anterior, SO soleus, MG medial gastrocnemius, VM vastus medialis, RF rectus femoris, ST semitendinosus, BF biceps femoris, GM gluteus medius (color figure online).

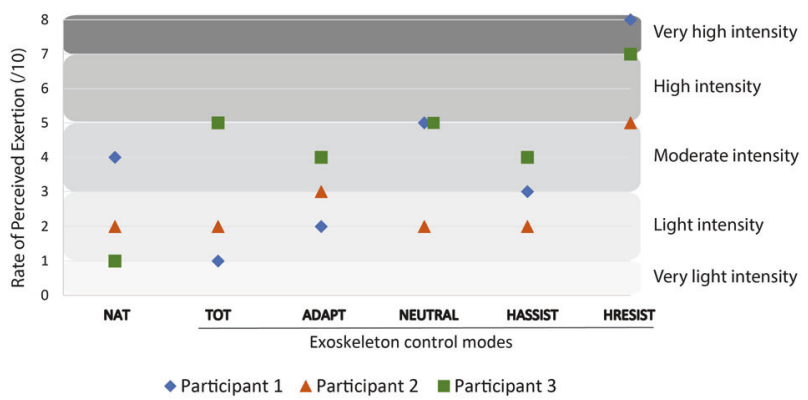

Fig. 4 Ratings of perceived effort during all walking trials for each participant. Areas highlighted in various shades of gray represent different exercise intensities (i.e., very light, light, moderate, vigorous, near maximum intensity, maximal, and sub-maximal effort) according to the ACSM's guidelines (color figure online).

into different muscle weightings found in able-bodied individuals.

This study provided new evidence that has the potential to impact clinical practice. First, although not all WRE control modes induced motor control adaptations closer to the able-bodied reference, the results showed that typical MS characteristics found in abled-bodied individuals during overground walking without a WRE could be reproduced when individuals with iSCI ambulate with WRE. These aspects might have important implications when selecting WRE control modes before engaging on locomotor training programs using this technology. Second, when exploring the level of effort required to walk during all WRE nontrajectory-controlled modes, the HASSIST mode required a light to moderate effort from all participants to accomplish the walking task. Thus, this control mode could be used to facilitate the swing phase during prolonged periods of walking (i.e., massed practice) and, ultimately, induce beneficial neural plasticity and potentiate locomotor recovery [18].

This study had several limitations. First, the small sample of adults with a chronic iSCI does not allow generalization of the results. In fact, other individuals may benefit from other WRE control modes. Second, since no kinematic analysis was completed in the present study, it remains difficult to determine to what extent WRE movement strategies were similar to those established for overground walking. Lastly, the actual absolute level of assistance or resistance provided remains unknown and is not provided by the WRE manufacturer.

\section{Conclusion}

Walking with a WRE in control modes allowing step variability (i.e., self-selected trajectory), and assisting L/E swing phase (i.e., HASSIST), best replicated MSs observed in ablebodied individuals during overground walking, while requiring light to moderate effort. This control mode may allow adults with iSCI to engage in a high-repetition task-specific walking program (i.e., activity-based therapy) needed to induce neuroplastic adaptations and potentiate walking ability. Additional studies with more robust experimental designs and larger sample sizes are needed to strengthen evidence and further support clinical decision-making processes when aiming to improve $\mathrm{L} / \mathrm{E}$ motor control during walking. Nonetheless, the results of the present study are a first step towards a better understanding of the effects of various control modes on L/E muscular coordination, which can be evaluated through MSs when individuals with iSCI walk with a WRE.

Acknowledgements The authors would like to acknowledge Philippe Gourdou and Martin Vermette, PT, for their assistance with data processing and project coordination, respectively.

Funding MJE was supported by a doctoral scholarship from the Fonds de Recherche du Québec-Santé (FRQ-S) and the Initiative for the development of new technologies and practices in rehabilitation (INSPIRE). DHG is supported by a senior research scholarship from the FRQ-S and co-leads the INSPIRE. The equipment used for this project was funded by a grant from the John-R.-Evans leaders program of the Canada Foundation for Innovation (\#36243).

\section{Compliance with ethical standards}

Conflict of interest The authors declare that they have no conflict of interest.

Publisher's note Springer Nature remains neutral with regard to jurisdictional claims in published maps and institutional affiliations.

\section{References}

1. Asselin PK, Avedissian M, Knezevic S, Kornfeld S, Spungen AM. Training persons with spinal cord injury to ambulate using a powered exoskeleton. J Vis Exp. 2016;112:e54071.

2. Morawietz C, Moffat F. Effects of locomotor training after incomplete spinal cord injury: a systematic review. Arch Phys Med Rehabilit. 2013;94:2297-308.

3. Swinnen E, Duerinck S, Baeyens J-P, Meeusen R, Kerckhofs E. Effectiveness of robot-assisted gait training in persons with spinal cord injury: a systematic review. J Rehabilit Med. 2010;42:520-6.

4. Lewek MD, Cruz TH, Moore JL, Roth HR, Dhaher YY, Hornby TG. Allowing intralimb kinematic variability during 
locomotor training poststroke improves kinematic consistency: a subgroup analysis from a randomized clinical trial. Phys Ther. 2009;89:829-39.

5. Cai LL, Fong AJ, Otoshi CK, Liang Y, Burdick JW, Roy RR, et al. Implications of assist-as-needed robotic step training after a complete spinal cord injury on intrinsic strategies of motor learning. J Neurosci. 2006;26:10564-8.

6. Clark DJ, Ting LH, Zajac FE, Neptune RR, Kautz SA. Merging of healthy motor modules predicts reduced locomotor performance and muscle coordination complexity post-stroke. J Neurophysiol. 2010;103:844-57.

7. Kibushi B, Hagio S, Moritani T, Kouzaki M. Speed-dependent modulation of muscle activity based on muscle synergies during treadmill walking. Front Hum Neurosci. 2018;12:4.

8. Hagio S, Kouzaki M. The flexible recruitment of muscle synergies depends on the required force-generating capability. J Neurophysiol. 2014;112:316-27.

9. Rimini D, Agostini V, Knaflitz M. Intra-subject consistency during locomotion: similarity in shared and subject-specific muscle synergies. Front Hum Neurosci. 2017;11:586.

10. Escalona MJ, Bourbonnais D, Le Flem D, Goyette M, Duclos C, Gagnon DH. Effects of diverse robotic exoskeleton control options on lower limb muscle synergies during overground walking in able-bodied adults. 2020. Manuscript submitted for publication.

11. Nishida K, Hagio S, Kibushi B, Moritani T, Kouzaki M. Comparison of muscle synergies for running between different foot strike patterns. PLoS ONE. 2017;12:e0171535.
12. Haley SM, Fragala-Pinkham MA. Interpreting change scores of tests and measures used in physical therapy. Phys Ther. 2006;86:735-43.

13. Riebe D, Ehrman JK, Liguori G, Magal M. ACSM's guidelines for exercise testing and prescription. 10th ed. In: Riebe D, Ehrman JK, Liguori G, Magal M, editors. Philadelphia, PA: Wolters Kluwer; 2017.

14. Escalona MJ, Brosseau R, Vermette M, Comtois AS, Duclos C, Aubertin-Leheudre M, et al. Cardiorespiratory demand and rate of perceived exertion during overground walking with a robotic exoskeleton in long-term manual wheelchair users with chronic spinal cord injury: A cross-sectional study. Ann Phys Rehabilit Med. 2018;61:215-23.

15. Hayes HB, Chvatal SA, French MA, Ting LH, Trumbower RD. Neuromuscular constraints on muscle coordination during overground walking in persons with chronic incomplete spinal cord injury. Clin Neurophysiol. 2014;125:2024-35.

16. Pérez-Nombela S, Barroso F, Torricelli D, de Los Reyes-Guzmán A, Del-Ama A, Gómez-Soriano J, et al. Modular control of gait after incomplete spinal cord injury: differences between sides. Spinal Cord. 2017;55:79-86.

17. Routson RL, Clark DJ, Bowden MG, Kautz SA, Neptune RR. The influence of locomotor rehabilitation on module quality and post-stroke hemiparetic walking performance. Gait Posture. 2013;38:511-7.

18. Harkema SJ. Neural plasticity after human spinal cord injury: application of locomotor training to the rehabilitation of walking. Neuroscientist. 2001;7:455-68. 